\title{
Reactive Laser Ablation Electrospray Ionization Time-Resolved Mass Spectrometry of Click Reactions
}

\author{
Fred A. M. G. van Geenen, ${ }^{\dagger, \ddagger \odot}$ Maurice C. R. Franssen, ${ }^{\dagger} \odot$ Han Zuilhof, ${ }^{\dagger, \S \odot}$
}

and Michel W. F. Nielen ${ }^{*},+\| \odot$

${ }^{\dagger}$ Laboratory of Organic Chemistry, Wageningen University, Stippeneng 4, 6708 WE Wageningen, The Netherlands

†TI-COAST, Science Park 904, 1098 XH Amsterdam, The Netherlands

${ }^{\S}$ School of Pharmaceutical Sciences and Technology, Tianjin University, 92 Weijin Road, Tianjin 300072, People’s Republic of China

"RIKILT, Wageningen University \& Research, P.O. Box 230, 6700 AE Wageningen, The Netherlands

Supporting Information

ABSTRACT: Reactions in confined compartments like charged microdroplets are of increasing interest, notably because of their substantially increased reaction rates. When combined with ambient ionization mass spectrometry (MS), reactions in charged microdroplets can be used to improve the detection of analytes or to study the molecular details of the reactions in real time. Here, we introduce a reactive laser ablation electrospray ionization (reactive LAESI) timeresolved mass spectrometry (TRMS) method to perform and study reactions in charged microdroplets. We demonstrate this approach with a class of reactions new to reactive ambient ionization MS: so-called click chemistry reactions. Click reactions are high-yielding reactions with a high atom efficiency, and are currently drawing significant attention from fields ranging from bioconjugation to polymer modification. Although click reactions are typically at least moderately fast (time scale of minutes to a few hours), in a reactive LAESI approach a substantial increase of reaction time is required for these reactions to occur. This increase was achieved using microdroplet chemistry and followed by MS using the insertion of a reaction tube- up to $1 \mathrm{~m}$ in length-between the LAESI source and the MS inlet, leading to near complete conversions due to significantly extended microdroplet lifetime. This novel approach allowed for the collection of kinetic data for a model (strain-promoted) click reaction between a substituted tetrazine and a strained alkyne and showed in addition excellent instrument stability, improved sensitivity, and applicability to other click reactions. Finally, the methodology was also demonstrated in a mass spectrometry imaging setting to show its feasibility in future imaging experiments.
$\mathrm{M}$ ass spectrometry (MS), stand-alone or hyphenated with separation techniques like chromatography or electrophoresis, is a well-established analysis technique in industry, healthcare, and many fields of science, such as organic synthesis, ${ }^{1}$ metabolomics, ${ }^{2}$ and proteomics. ${ }^{3}$ The abundant use of MS and its hyphenations is mainly due to the combination of high sensitivity, selectivity, speed, and capabilities for structure elucidation by $\mathrm{MS}^{n}$. MS can also be optimized to study molecular reactions in solution. ${ }^{4}$ Timeresolved mass spectrometry (TRMS), where reaction vessels are directly coupled with MS analyses, was first introduced by Lee et al. in 1989. ${ }^{5}$ TRMS is used to study dynamic processes in which reagents convert into (by-)products in real time, typically in the microsecond to minute time range. ${ }^{6-12}$ The development and use of TRMS has been reviewed recently by Lento and Wilson. ${ }^{13}$

The discovery of reaction acceleration in charged microdroplets, even up to 6 orders of magnitude, ${ }^{14}$ by the Cooks and
Zare groups brings a new way to perform and study a wide range of (bio)molecular reactions. ${ }^{15,16}$ It has been used in organic synthesis, ${ }^{17-20}$ in order to predict the likely success of up-scaling reactions, ${ }^{21}$ and as a continuous synthesis system. ${ }^{22}$ Other exciting fields of application are its use to study the chemistry of life, ${ }^{23}$ which mostly takes place in small enclosed volumes such as cells or atmospheric aerosols, ${ }^{24-26}$ or to improve detection of analytes in ambient ionization MS. ${ }^{27-32}$ Several explanations have been suggested why reaction acceleration in charged microdroplets may occur: partial desolvation of reagents at the droplet-to-air interface, ${ }^{32-37}$ $\mathrm{pH}$ change, ${ }^{38}$ and/or a potentially increased reagent concentration associated with solvent evaporation. ${ }^{20}$

Received: May 23, 2018

Accepted: July 31, 2018

Published: July 31, 2018 
The combination of TRMS and charged microdroplets can also be used to determine reaction kinetics, provided that reaction times can be accurately measured. Previously, reaction times with electrospray ionization (ESI) over distances up to $100 \mathrm{~cm}$ were estimations based on approximate droplet flight time, assuming that droplets are carried along by the nebulizing gas. ${ }^{39}$ Further steps have been taken to record reaction time with desorption electrospray ionization (DESI) ${ }^{40}$ and microdroplet fusion in extractive electrospray ionization $(\mathrm{EESI})^{41}$ by using Doppler techniques to measure the droplet velocity on the microsecond time scale. ${ }^{42,43}$ With DESI the droplet velocity was decreasing quickly with distance due to aerodynamic drag. ${ }^{42}$ With EESI, pressurized nebulizing nitrogen gas was used as a propulsive force providing more stable average velocities before reaching the MS. ${ }^{43}$ The reaction is essentially stopped when the droplets reach the vacuum of the heated MS interface. ${ }^{39} \mathrm{Up}$ to now, a direct way to measure reaction time over distances up to $100 \mathrm{~cm}$ has, to our knowledge, not been described.

Laser ablation electrospray ionization (LAESI) MS provides a way to analyze a wide range of samples, including liquids, ${ }^{44,45}$ solid materials, ${ }^{46}$ tissue, ${ }^{47-51}$ and single cells ${ }^{52-54}$, under ambient conditions. ${ }^{55}$ In LAESI, a pulsed laser directed onto a substrate produces a neutral plume of ablated material, which can be extracted with charged microdroplets produced by an electrospray probe for subsequent analyte ionization. When the electrospray solution contains a reagent, LAESI can be used to perform reactions with the ablated compounds in charged microdroplets. A combination with TRMS would enable the determination of reaction kinetics. Alternatively, the reaction could be used to generate chemical derivatives of the ablated compounds to improve the detection of analytes in ambient ionization MS. So far, mainly reactive DESI has been used in order to improve analyte detection by increased proton affinity, ${ }^{28-32}$ adapted analyte mass-to-charge $(\mathrm{m} / z)$ value $^{56-60}$ altered polarity, ${ }^{61-63}$ or as a tool for structure elucidation. ${ }^{64}$ Apart from DESI also a few other reactive ambient ionization techniques have been reported. ${ }^{65-69}$ Potentially, similar reagents can also be used in a reactive LAESI system. In current literature, however, reactive LAESI exclusively refers to coordination complexes with metal salts like lithium or silver in order to achieve cationization. ${ }^{70,71} \mathrm{~A}$ reason why common DESI reactions have not been reported for LAESI could be the relatively short reaction time window available in LAESI. For a comparable extractive electrospray ionization setup, the reaction time was stated to be in the order of only $15 \mu \mathrm{s} .{ }^{43}$ Thus, a major challenge for molecular reactions in ambient ionization LAESI MS is to significantly increase the reaction time. One possibility to achieve this is the introduction of a reaction tube, as previously used in an ESI study of a Hantzsch reaction. ${ }^{39}$ However, for introduction of a reaction tube into a reactive LAESI setting additional space needs to be available between the electrospray probe and reaction tube in order to allow the laser to irradiate the sample, ablate the material, and subsequently extract the plume by electrospray-generated charged microdroplets. In this experimental setup, temporal resolution can then be obtained in a direct and precise fashion by the time difference between the laser pulse and the MS detection of analytes or reaction products.

In the present work we report a novel ambient ionization MS approach, viz., reactive LAESI in combination with accurate reaction time determination. The method is demonstrated with an inverse electron demand Diels-Alder addition (IEDDA) - click-reaction between substituted tetrazines and a strained alkyne or alkene to form a stable pyridazine product. As a result of the insertion of a reaction tube, the (charged) microdroplet lifetime, and thus reaction time, was increased up to $4-5$ orders of magnitude. Two new chemical bonds are formed in this reaction, underlining the progress versus the cationization LAESI experiments mentioned above. Click reactions are drawing significant attention from different fields such as labeling of biomolecules for imaging, ${ }^{72,73}$ bioconjugation, ${ }^{74-77}$ and polymer modification, ${ }^{78}$ due to the high efficiency. ${ }^{79,80}$ Our reactive LAESI methodology successfully showed pseudo-first-order kinetic data of a model click reaction system between $\mathrm{BCN}$-amine $\mathbf{1}$ and dipyridyl-tetrazine $\mathbf{2 a}$, whereas TCO-amine $\mathbf{4}$ and other tetrazines performed equally well (structures provided in Figure 1). Finally, the feasibility of reactive LAESI mass
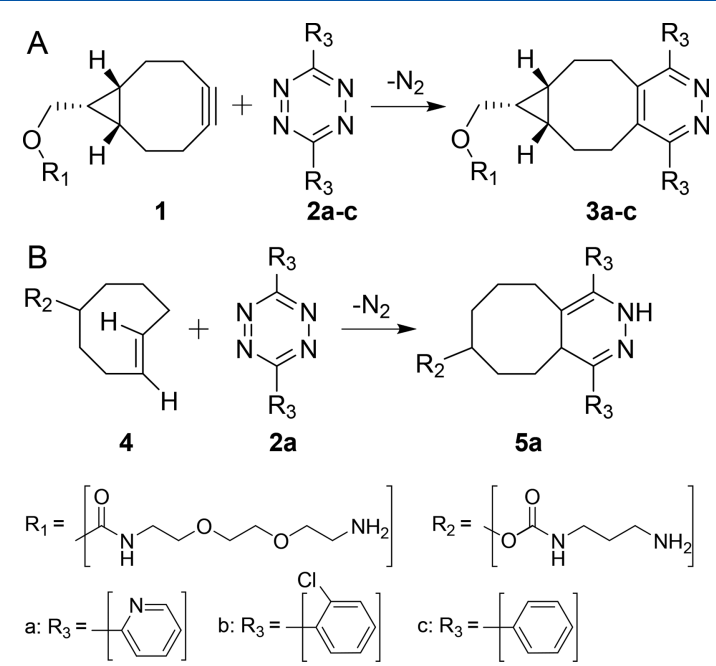

Figure 1. IEDDA reactions between an amine-terminated strained (A) cyclooctyne (BCN-amine; 1) or (B) cyclooctene (TCO-amine; 4) and disubstituted tetrazines 2 which feature different polarities (dipyridyl-tetrazine $\mathbf{2 a}$, clofentezine $\mathbf{2 b}$, diphenyl-tetrazine $\mathbf{2 c}$ ). The $[4+2]$ cycloaddition initially forms a strained bicyclic reaction intermediate that is converted upon release of $\mathrm{N}_{2}$ into the pyridazine 3 or dihydropyridazine 5 click reaction product.

spectrometry imaging (MSI) was demonstrated by exploiting the combined potential of laser spatial resolution and microdroplet-induced reaction acceleration.

\section{EXPERIMENTAL SECTION}

Materials. Ultrapure water $\left(\mathrm{H}_{2} \mathrm{O}\right), 18.2 \mathrm{M} \Omega \mathrm{cm}^{-1}$ at 25 ${ }^{\circ} \mathrm{C}$, was freshly produced daily with a Millipore (Molsheim, France) Integral 3 system. Methanol ( $\mathrm{MeOH}), \mathrm{LC}-\mathrm{MS}$ grade, was purchased from VWR (Leuven, Belgium). $N-[(1 R, 8 S, 9 s)$ Bicyclo[6.1.0]non-4-yn-9-ylmethyloxycarbonyl]-1,8-diamino3,6-dioxaoctane (BCN-amine, 1), trans-4-cycloocten-1-yl(3aminopropyl) carbamate (TCO-amine, 4), 3,6-di-2-pyridyl1,2,4,5-tetrazine (dipyridyl-tetrazine, 2a), 3,6-di-2-chlorophenyl-1,2,4,5-tetrazine (clofentezine, 2b), 3,6-diphenyl-1,2,4,5tetrazine (diphenyl-tetrazine, $2 \mathrm{c}$ ), reserpine, and 96-well plates (untreated) were obtained from Sigma-Aldrich (Zwijndrecht, The Netherlands). Chemical structures of reagents are given in Figure 1. All chemicals were used without further purification.

Experimental Setup and Measurement of Reaction Time. A Protea Biosciences (Morgantown, WV) LAESI DP- 

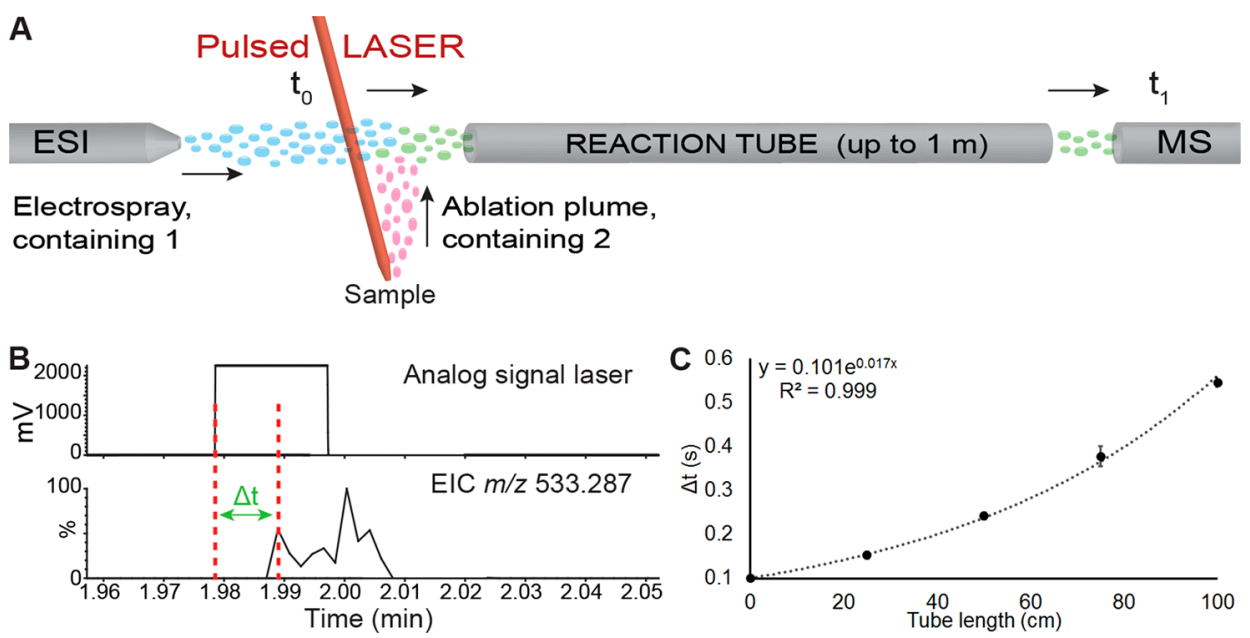

Figure 2. (A) Overview of reactive LAESI setup as used, comprising a pneumatically assisted ESI probe containing click reagent 1 , a well plate (not shown) containing click reagent 2, a mid-IR laser, and a metal reaction tube $(0-100 \mathrm{~cm})$. Other details can be found in the Experimental Section and the Supporting Information. (B) Specification of reaction time (start time laser pulse - time first detection event of product 3a) with (upper trace) the analog signal of the laser pulse and (lower trace) the detection of click reaction product $3 a, m / z 533.287$. (C) Graph of reaction time vs reaction tube length $(n=2)$.

1000 system was electronically connected to a Waters (Manchester, U.K.) Synapt G2-S traveling wave ion mobility time-of-flight mass spectrometer and used for all analyses. The distance between the LAESI source and the MS inlet was varied between 1 and $100 \mathrm{~cm}$ (see Figure 2A). Transport of ions from the LAESI ionization source to the MS inlet was assisted by nitrogen as electrospray nebulizer gas, controlled at $1 \mathrm{~L} \mathrm{~min}^{-1}$ by a Bronkhorst (Veenendaal, The Netherlands) Mani-Flow mass flow controller, directed toward the inlet of a grounded copper reaction tube of $1-100 \mathrm{~cm}$ length and internal diameter of $1.0 \mathrm{~cm}$ (Gamma, The Netherlands) at room temperature. The nebulizer gas flow was optimized for $50 \mathrm{~cm}$ reaction tube length. Distances between the ESI to the reaction tube and the reaction tube to the MS inlet, both under ambient conditions, were 18 and $20 \mathrm{~mm}$, respectively. A picture of the experimental setup is shown in Figure S1. LAESI desktop software v.2.0.1.3 (Protea Biosciences) was used to control experimental parameters of the LAESI system. The $\mathrm{Nd}$ :YAG optical parametric oscillator mid-infrared laser (2.94 $\mu \mathrm{m})$ was set to $100 \%$ laser power $\left(\Phi 3.2 \mathrm{~J} / \mathrm{cm}^{2}\right)$, and 10 pulses with a specified pulse length of $5 \mathrm{~ns}$ were acquired on every spot on the 96-well plate at a frequency of $10 \mathrm{~Hz}$. The 96-well plate contained $383 \mu \mathrm{L}$ of $100 \mu \mathrm{M}$ dipyridyl-tetrazine $2 \mathrm{a}$ in $\mathrm{MeOH}-\mathrm{H}_{2} \mathrm{O}$ (1:1) per well, and the temperature-controlled sample stage was cooled to $4{ }^{\circ} \mathrm{C}$ to avoid evaporation during analysis. A solution of $100 \mu \mathrm{M} \mathrm{BCN}$-amine 1 in $\mathrm{MeOH}-\mathrm{H}_{2} \mathrm{O}$ (1:1) was used as electrospray solvent at a flow rate of $5 \mu \mathrm{L} /$ min. Electrospray voltage was set at $\sim 3.5 \mathrm{kV}$ in order to have a stable Taylor cone. The Synapt G2-S was controlled by MassLynx v4.1 SCN 883 (Waters) and operated in positiveion time-of-flight (TOF)-MS resolution mode, $\mathrm{m} / z$ range of 50-1200 Da, scan time of $0.1 \mathrm{~s}$, source and interface temperatures both set at $120{ }^{\circ} \mathrm{C}$, sample cone $20 \mathrm{~V}$, source offset $60 \mathrm{~V}$, automated detector check off, pDRE lens 99.80\%, and detector value set to an additional $+100 \mathrm{~V}$. In addition, the trap gas was switched from default argon to nitrogen operated at a flow rate of $2 \mathrm{~mL} / \mathrm{min}$, the helium cell dc was set to $0.0 \mathrm{~V}$, and the trap and transfer collision energies were at $1.0 \mathrm{~V}$ to reduce any ion fragmentation in the mass spectrometer. Reaction time $\Delta t$ was specified as the time difference between the analog signal of the laser pulse and the first mass spectrometric detection event of the reaction product $3 \mathbf{a}$ (Figure 2B). Different reaction tube lengths, 0, 25, 50, 75, and $100 \mathrm{~cm}$, were used to obtain different reaction times. The $y$ axis cutoff in the measurement of reaction time graphs (Figure $2 \mathrm{C}$ and Figure S2) was set to $\Delta t=0.10 \mathrm{~s}$ in order to allow exponential fitting. In subsequent calculations on reaction time the added value of $\Delta t=0.10 \mathrm{~s}$ was subtracted. Where relevant in figures, the standard deviation of averaged data is provided as error bars.

Determination of Reaction Kinetics. The experiments that were used to determine the reaction time-described in the previous section-were optimized to achieve maximum temporal resolution, at the cost of signal intensity, with a short MS scan time and few laser pulses per analysis location. The reaction kinetics experiments required larger peak areas for data processing and were optimized for improved signal intensity. For this reason a few acquisition parameters were adjusted from the previous section. The MS scan time was set at $1 \mathrm{~s}$, and 75 laser pulses were used per analysis location to obtain significant reaction product peak areas for reliable integration in the extracted ion currents (EIC) obtained. Reaction time data were now simply calculated for reaction tube lengths of $0,1,2,3,4,5,10,15,20,25,50,75$, and 100 $\mathrm{cm}$, using the exponential fit $\left(y=0.101 \mathrm{e}^{0.017 x}\right)$ of the calibration plot given in Figure 2C. Raw MS data were processed to yield EICs with a $15 \mathrm{ppm}$ window. These ion currents were mean-smoothed (number of smooths was 1 , with a window size of 2 scans) and integrated to obtain laser pulse based peak areas. With the kinetic data (as presented in Figure 4, $n=3$ ), one outlier at time $19 \mathrm{~ms}$ was removed before averaging with a Grubbs' test based on residuals from the regression line (95\% CI, $10 \mathrm{DF}$ ). Background-subtracted mass spectra were created using the "combine spectrum" function in MassLynx: five scans, each corresponding to 10 laser pulses were averaged, and 50 scans of the electrospray background were subtracted.

Reactive LAESI Mass Spectrometry Imaging of the Wageningen University (WUR) Logo. For mass spectrometry imaging the same experimental conditions were used as 
described in the Determination of Reaction Kinetics section, with a few changes. The reaction tube length was fixed at 50 $\mathrm{cm}$, MS inlet and source temperatures were at $150{ }^{\circ} \mathrm{C}, 20$ laser pulses were used per $x-y$ position with a between location dwell time of $0 \mathrm{~s}$, and the LAESI sample stage was cooled further down to $-19{ }^{\circ} \mathrm{C}$ to keep the sample frozen during the experiment. Reactive LAESI data were acquired from a $61 \times 41$ pattern (2501 sample locations) - laser spot size approximately $200 \mu \mathrm{m}$, in-between spot interval $500 \mu \mathrm{m}-$ in a sampling area defined from an optical image, resulting in a total analysis time of $180 \mathrm{~min}$. The sample was created by depositing $200 \mu \mathrm{L}$ of a $1 \mathrm{mM}$ dipyridyl-tetrazine 2 a solution in $\mathrm{H}_{2} \mathrm{O}$ on the hydrophilic part (in the shape of the Wageningen University logo) of an otherwise hydrophobic surface. The sample was then stored in a freezer at $-18^{\circ} \mathrm{C}$ for $30 \mathrm{~min}$ in order to freeze the aqueous solution prior to MSI analysis.

Safety Precautions. Safety precaution: since the metal reaction tube is in close proximity with the electrospray needle $(\sim 3.5 \mathrm{kV})$, there is risk of electric discharge when not either decently grounded or covered by a nonconductive material. In addition, the open gap between the reaction tube and the MS inlet could contain solvent vapor; thus, a suction hood is required to remove any harmful solvent vapors that are not sucked into the MS inlet. Finally, there is an open heated MS inlet, which constitutes a potential temperature hazard.

\section{RESULTS AND DISCUSSION}

Measurement of Click Reaction Time. In order to study the reaction time in the click reaction between strained alkyne $\mathbf{1}$ and tetrazine $\mathbf{2 a}$, the following setup was made. A pulsed mid-IR laser $(2.94 \mu \mathrm{m})$ was used to ablate a dipyridyl-tetrazine 2a solution. The ablation plumes were continuously extracted with orthogonally directed electrospray microdroplets containing $\mathrm{BCN}$-amine 1 reagent. This aerosol is carried along with nebulizer gas through a metal tube before MS detection. To obtain different reaction times, several metal tubes of different lengths $(0,25,50,75$, and $100 \mathrm{~cm})$ were used. A schematic overview is presented in Figure 2A, and an image of the setup is shown Figure $S 1$. The time difference between the laser pulse and the MS detection of product 3a (Figure 2B) is plotted against increasing tube lengths in Figure 2C. After an initial relatively high linear gas velocity due to the small diameter of nebulizer gas outlet, the velocity is rapidly decreasing, which yields for this range of tube lengths a near-exponential dependence of reaction times on reaction tube length $\left(R^{2}=0.999\right)$. This correlation could therefore be used as a calibration curve for other tube lengths up to $100 \mathrm{~cm}$. The electrical voltage offset between the MS inlet and grounded tube was varied to investigate whether charge attraction/repulsion would affect the reaction time; yet, no significant effect was observed (Figure S2).

Figure $3 \mathrm{~A}$ presents a background-subtracted mass spectrum for the reaction between $\mathrm{BCN}$-amine $\mathbf{1}$ and dipyridyl-tetrazine 2a after $140 \mathrm{~ms}$ of reaction time. Three $\mathrm{m} / z$ values are observed: protonated dipyridyl-tetrazine 2 a at $\mathrm{m} / z$ 237.086, protonated product $3 \mathrm{a}$ at $\mathrm{m} / \mathrm{z} 533.289$, and $\mathrm{m} / \mathrm{z}$ 495.148, which corresponds to the sodium adduct of dipyridyl-tetrazine dimer. In order to further demonstrate this methodology, a different click reaction dienophile, TCO-amine 4, was also used as electrospray additive for the reaction with dipyridyltetrazine 2a. The obtained background-subtracted mass spectrum is shown in Figure 3B. Like Figure 3A, both
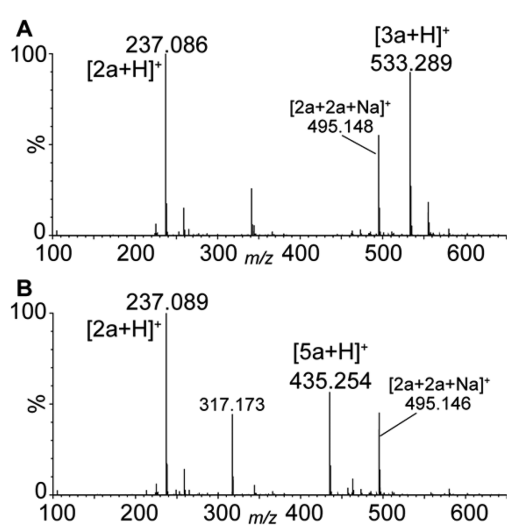

Figure 3. Background-subtracted reactive LAESI mass spectra of the click reaction products between (A) $\mathrm{BCN}$-amine 1 and dipyridyltetrazine $\mathbf{2 a}$ and (B) TCO-amine $\mathbf{4}$ and dipyridyl-tetrazine $\mathbf{2 a}$, following the setup as depicted in Figure 2 (tube length $=50 \mathrm{~cm}$ ).

protonated dipyridyl-tetrazine $\mathbf{2} \mathbf{a}$ and its sodium adduct dimer were detected at $m / z 237.089$ and 495.146, respectively. The reaction product $5 \mathbf{a}$ is detected at $m / z$ 435.254, demonstrating the formation of covalently bonded reaction products in reactive LAESI MS. Furthermore, $\mathrm{m} / z$ value 317.173 was obtained, which is likely to be formed by elimination of the $R_{2}$ group of structure $\mathbf{5}$ in Figure 1 ; the proposed structure is presented in Figure $S 3$ (note: the truncated $R_{2}$ group is already present in $\mathbf{4}$ as an impurity, see Figure S4). Any effect of the nature of the metal from the reaction tube on the reaction was briefly examined by using both copper and stainless steel tubes, and no difference in reaction was observed (Figure S5).

When Figure $3 \mathrm{~A}$ and Figure S5B are compared, a few differences stand out: in Figure $3 \mathrm{~A}$ both tetrazine $2 \mathrm{a}$ and its dimer are well-depicted as a result of the relatively high (1 $\mathrm{mM}$ ) tetrazine $\mathbf{2 a}$ concentration, whereas in Figure S5B (100 $\mu \mathrm{M}$ tetrazine 2 a concentration) $\mathrm{m} / z$ value 561.296 is observed; this ion was found to be an adduct between both click reagents (Figure S6), likely due to H-bonding between the protonated amine on 1 and the pyridine ring on $2 \mathrm{a} . \mathrm{m} / z 561.296$ is absent in Figure $3 \mathrm{~A}$ due to the higher MS source temperature used $\left(150{ }^{\circ} \mathrm{C}\right.$ instead of $\left.120^{\circ} \mathrm{C}\right)$, effectively breaking the supposed $\mathrm{H}$-bond prior to mass analysis.

Finally, the solvent droplet lifetime over the $0.5 \mathrm{~s}(100 \mathrm{~cm})$ experimental time frame was indirectly investigated by following the area ratio between reaction product $3 \mathbf{a}$ and its $\left[3 \mathbf{a}+\mathrm{H}_{2} \mathrm{O}\right]$ adduct ions. Tabulating these ratios for multiple tube lengths (Table S7) results in a steady ratio increase corresponding with increasing tube length. This data suggests gradual droplet evaporation inside our reaction tube, but the microdroplets do not seem to completely evaporate even at distances up to $100 \mathrm{~cm}$, as the $\mathrm{H}_{2} \mathrm{O}$ adduct is still detected here (Figure S8). This is in accordance with earlier reported results by Lee et al., ${ }^{43}$ where the evaporation of water microdroplets produced by an electrospray probe was found to be negligible, although in a much shorter time frame of $50 \mu \mathrm{s}$.

Determination of Model Click Reaction System Kinetics. Following the time measurements and detection of click reaction products $\mathbf{3 a}$ and $\mathbf{5} \mathbf{a}$, which are both only possible due to the insertion of a reaction tube, the TRMS method was used to study the IEDDA reaction kinetics in charged microdroplets under pseudo-first-order conditions. The latter was achieved with an excess of $\mathrm{BCN}$-amine 1 , as the concentration in the electrospray microdroplets is much larger 
than the dipyridyl-tetrazine $\mathbf{2 a}$ in the ablated liquid solution (Figure S9). Reserpine was added as internal standard to the dipyridyl-tetrazine 2 a solution in order to correct for possible ablation variation and any bias caused by changing the distance between the electrospray source and MS inlet via stepwise variation of the reaction tube length (13 different tube lengths were used). The product $\mathbf{3 a}$ formation over time is presented in Figure 4, from which the pseudo-first-order rate constant for

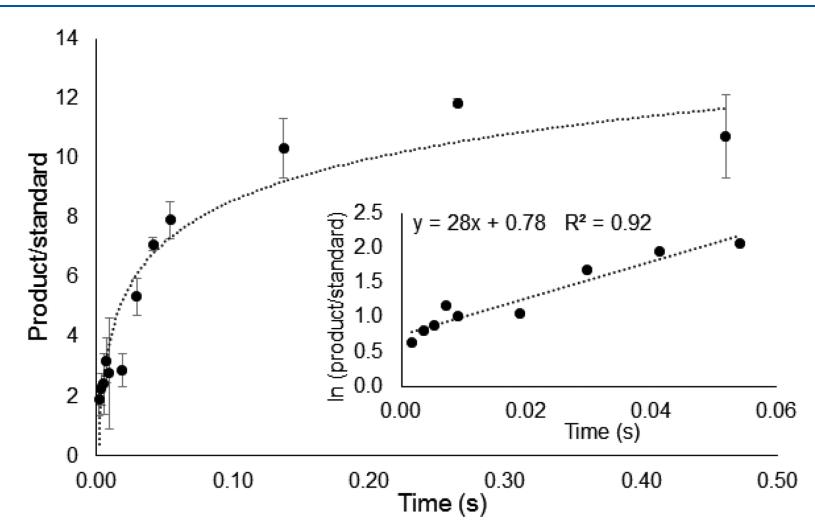

Figure 4. Graph of the formation of click reaction product $3 \mathbf{a}$ in relation to reaction time $(n=3)$ following insertion of 13 different reaction tube lengths $(0-100 \mathrm{~cm})$ for the model reaction between $\mathrm{BCN}$-amine $\mathbf{1}$ and dipyridyl-tetrazine $\mathbf{2 a}$. Inserted is the graph with the $\ln$ of data points up to $60 \mathrm{~ms}$.

the IEDDA reaction was determined to be $28 \mathrm{~s}^{-1}$. One additional interesting finding was that the absolute peak areas of both click reaction product $3 \mathbf{a}$ and the used standard reserpine were surprisingly stable over reaction tube lengths longer than $15 \mathrm{~cm}$, indicating that almost no signal was lost upon increasing the reaction tube length even up to $1 \mathrm{~m}$, corresponding to a $0.5 \mathrm{~s}$ reaction time (Figure S10).

The second-order rate constant $k_{2}$ is then derived as $28 \mathrm{~s}^{-1} /$ $0.10 \mathrm{mM}=2.8 \times 10^{5} \mathrm{M}^{-1} \mathrm{~s}^{-1}$. The analogous reaction between dipyridyl-tetrazine $2 \mathrm{a}$ and $\mathrm{BCN}-\mathrm{OH}$ performed in methanol solution was reported in various studies to have a $k_{2}$ of typically $10^{2} \mathrm{M}^{-1} \mathrm{~s}^{-1} \cdot{ }^{74,75}$ The current reaction in charged microdroplets is at least 2 orders of magnitude faster than the similar click reaction in bulk solvents. It has been reported that the addition of water as cosolvent $\left(55: 45 \mathrm{MeOH}-\mathrm{H}_{2} \mathrm{O}\right)$ increases the rate constant due to hydrogen bonding with tetrazine, which reduces the highest occupied molecular orbital-lowest unoccupied molecular orbital (HOMO-LUMO) gap. ${ }^{76}$ The effect of a decreasing $\mathrm{pH}$ could also contribute to the increased reaction rate because of possible protonation of the pyridine ring, thus increasing its electron-withdrawing properties and concomitantly speeding up the reaction. Finally, evaporation of solvent molecules could also cause the reaction rate to increase as a result of increasing concentration; however, solvent droplets do not seem to fully evaporate before entering the vacuum of the MS inlet, as discussed above. It is therefore likely that the observed reaction acceleration is due to a combination of effects originating from the charged microdroplet environment, as discussed by Yan et al. ${ }^{81}$

Reactive LAESI Robustness and Scope. The robustness of the methodology and its application to various tetrazines with different polarities was briefly investigated using clofentezine $\mathbf{2 b}$ and diphenyl-tetrazine $\mathbf{2 c}$. For this, four rows of a 96-well plate were filled with different tetrazine solutions per row; from row A to D: blank, dipyridyl-tetrazine 2a, clofentezine $\mathbf{2 b}$, and diphenyl-tetrazine $\mathbf{2 c}$, all $1 \mathrm{mM}$ in $\mathrm{MeOH}-\mathrm{H}_{2} \mathrm{O}$ (95:5). Subsequently, the analysis was run starting at row $\mathrm{A}$ and followed a typewriter pattern to end in row D. Unprocessed EICs and the analog signal of the laserpulsed injection of tetrazines are shown in Figure 5.

In the blank, at 1-4 min, no signal was obtained in the EIC of any $\mathrm{BCN}$-amine 1 -tetrazine products. The click reaction product ions of diphenyl-tetrazine $3 \mathrm{c}$ (Figure 5A), clofentezine $3 \mathbf{b}$ (Figure 5B), and dipyridyl-tetrazine 3a (Figure 5C) were in excellent agreement with the time frame of the analog signal of ablation from the corresponding tetrazine. Although not studied, other (asymmetrical) tetrazines are expected to work as well. The EICs in Figure 5 also demonstrate that well-towell signal intensity stability is at least equal to or even better than that of conventional LAESI experiments (Figure S12), showing that ambient mass spectrometry imaging with reactive LAESI would be realistic.

Thanks to reactive LAESI, the sensitivity could be increased because of a higher ionization efficiency of the click reaction product $\mathbf{3 a}$ in comparison with dipyridyl-tetrazine $\mathbf{2 a}$. This effect of increased sensitivity combined with indications of the scope of reactive LAESI are shown in Figure 6, for which dipyridyl-tetrazine $2 \mathrm{a}$ was dissolved in both $\mathrm{H}_{2} \mathrm{O}$ and in 10

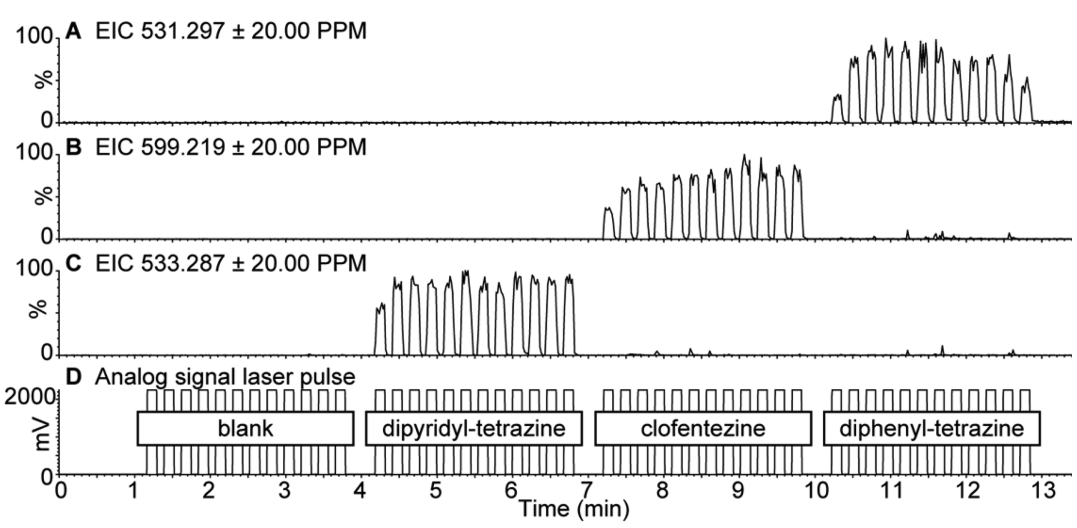

Figure 5. Reactive LAESI MS extracted ion currents of the click reaction products of 1 with (A) diphenyl-tetrazine 3c $(\mathrm{m} / z$ 531.297), (B) clofentezine $3 \mathbf{b}(\mathrm{m} / \boldsymbol{z} 599.219)$, and $(\mathrm{C})$ dipyridyl-tetrazine 3a $(\mathrm{m} / z$ 533.287). Corresponding background-subtracted mass spectra are given in Figure S11, parts A and B, and Figure 3A, respectively. In addition, the analog signal of the laser pulses is displayed (D). The reaction tube length was $50 \mathrm{~cm}$. 


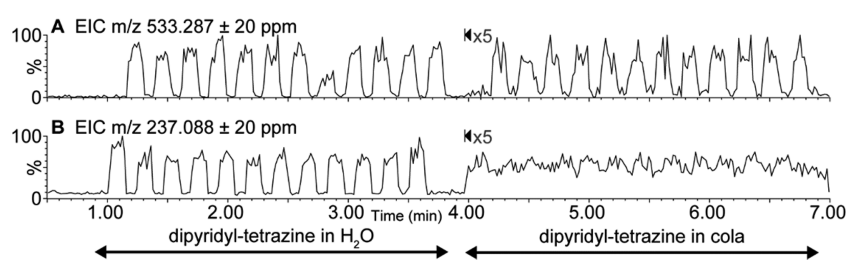

Figure 6. Reactive LAESI extracted ion currents of two separate analyses of a $100 \mu \mathrm{M}$ dipyridyl-tetrazine 2 a solution in $\mathrm{H}_{2} \mathrm{O}$ and in 10 times diluted cola. Analysis A was with $\mathrm{BCN}$-amine $\mathbf{1}$ in the electrospray solution and depicts the pyridazine product $3 \mathbf{a}(\mathrm{m} / \mathrm{z}$ 533.287) at all times. Analysis B was without BCN-amine 1 in the electrospray solution and shows dipyridyl-tetrazine $2 \mathbf{a}(\mathrm{m} / z$ 237.088) in a water environment but not in cola.

times diluted soft drink (cola) at a concentration of $100 \mu \mathrm{M}$ and analyzed with (Figure 6A) and without (Figure 6B) BCNamine 1 in the ESI spray solution. As a result, the click reaction product $3 \mathrm{a}$ could still be detected in diluted cola, whereas unreacted dipyridyl-tetrazine $\mathbf{2 a}$ got lost in the background.

Reactive LAESI Mass Spectrometry Imaging. Finally, preliminary reactive LAESI mass spectrometry imaging (MSI) experiments were conducted on a dipyridyl-tetrazine 2a solution which was deposited-and subsequently frozenonto a hydrophilic surface area in the shape of the Wageningen University logo (Figure S13; size $20 \mathrm{~mm} \times 29 \mathrm{~mm}$ ). An image was created by plotting the absolute intensity of the protonated click reaction product $3 \mathbf{a}$ according to laser ablation $x-y$ coordinates. The resulting 2D ion map nicely correlates with the sample (Figure 7), showing the potential to map the presence of compounds onto a surface using reactive LAESI MSI.

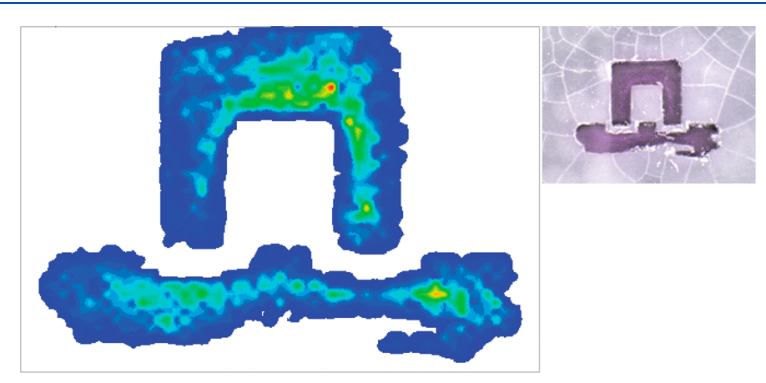

Figure 7. Reactive LAESI-MSI ion map of the Wageningen University logo via $500 \mu \mathrm{m}$ spatial resolution-see the Experimental Section-of $m / z 533.287 \pm 0.01 \mathrm{Da}$, being the click reaction product $3 \mathrm{a}[\mathrm{M}+$ $\mathrm{H}]^{+}$.

\section{CONCLUSIONS}

In conclusion, we have developed a novel ambient ionization time-resolved mass spectrometry method for performing and studying reactions in charged microdroplets. For this we developed a setup to allow the first reactive LAESI analysis with covalent bond-forming (click) reactions. The observation of the click reaction products was a result of the substantially increased reaction time due to the insertion of a reaction tube. Following a calibration of the exponential relationship between reaction time and reaction tube length using the time difference between the laser pulse and MS detection signals, we were able to determine kinetic data for the model system IEDDA (click) reaction between a tetrazine with a strained cycloalkyne. Additionally, this method has shown excellent stability and improved sensitivity and proved to be viable for several click reactions. With this reactive LAESI method, a wide range of molecular reactions can potentially be studied, to investigate rates, solvent effects on molecular reactions, or as a tool to improve the detection of target analytes in ambient ionization MS(I). Following these encouraging results, this reactive LAESI method could also be extended to other reactions previously reported in reactive DESI, like in the analyses of, e.g., saccharides with boronic acids, ${ }^{62}$ steroids with hydroxylamine, ${ }^{31}$ and/or protein-ligand interactions. ${ }^{82}$ Eventually, the present work is expected to enable in situ reactions during bioanalytical MSI.

\section{ASSOCIATED CONTENT}

\section{Supporting Information}

The Supporting Information is available free of charge on the ACS Publications website at DOI: 10.1021/acs.analchem. 8 b02290.

Picture of the reactive LAESI instrumental setup, graph of reaction time vs tube length, proposed structure of the compound having $m / z 317.173,{ }^{1} \mathrm{H}$ NMR spectrum of TCO-amine 4, background-subtracted mass spectra with copper and stainless steel reaction tubes, MS/MS mass spectrum of $m / z 561.3$, table and EICs of product 3a and its corresponding water adduct, EICs of product 3a and $\mathrm{BCN}$-amine 1 , graphs of absolute peak areas of product $3 \mathrm{a}$ and $\mathbf{1}$ /reserpine, reactive LAESI backgroundsubtracted mass spectra of reaction products of clofentezine $\mathbf{3 b}$ and diphenyl-tetrazine $3 \mathbf{c}$, EIC of $\mathbf{2 a}$ in regular LAESI conditions, and template of the Wageningen University logo (PDF)

\section{AUTHOR INFORMATION}

\section{Corresponding Author}

*Phone: +31 317 481784. E-mail: Michel.Nielen@wur.nl. ORCID

Fred A. M. G. van Geenen: 0000-0002-4284-6333

Maurice C. R. Franssen: 0000-0002-3615-9115

Han Zuilhof: 0000-0001-5773-8506

Michel W. F. Nielen: 0000-0003-4634-0249

\section{Author Contributions}

The manuscript was written through contributions of all authors. All authors have given approval to the final version of the manuscript.

\section{Notes}

The authors declare no competing financial interest.

\section{ACKNOWLEDGMENTS}

The authors thank Frank Claassen and Jan Commandeur for technical assistance, Sevil Sahin for the NMR measurement, and Martine Keijzer for preliminary experiments. The authors are grateful to receive funding for this research from The Netherlands Organization for Scientific Research (NWO) in the framework of the Technology Area TA-COAST2 of the Fund New Chemical Innovations (project no. 053.21.111).

\section{REFERENCES}

(1) Biemann, K. Angew. Chem., Int. Ed. Engl. 1962, 1, 98-111.

(2) Petras, D.; Jarmusch, A. K.; Dorrestein, P. C. Curr. Opin. Chem. Biol. 2017, 36, 24-31.

(3) Aebersold, R.; Mann, M. Nature 2016, 537, 347. 
(4) Ingram, A. J.; Boeser, C. L.; Zare, R. N. Chem. Sci. 2016, 7, 3955

(5) Lee, E. D.; Mueck, W.; Henion, J. D.; Covey, T. R. J. Am. Chem. Soc. 1989, 111, 4600-4604.

(6) Rob, T.; Wilson, D. J. J. Am. Soc. Mass Spectrom. 2009, 20, 124130.

(7) Pan, J.; Rintala-Dempsey, A. C.; Li, Y.; Shaw, G. S.; Konermann, L. Biochemistry 2006, 45, 3005-3013.

(8) Liuni, P.; Olkhov-Mitsel, E.; Orellana, A.; Wilson, D. J. Anal. Chem. 2013, 85, 3758-3764.

(9) Mortensen, D. N.; Williams, E. R. J. Am. Chem. Soc. 2016, 138, $3453-3460$.

(10) Jacobs, M. I.; Davies, J. F.; Lee, L.; Davis, R. D.; Houle, F.; Wilson, K. R. Anal. Chem. 2017, 89, 12511-12519.

(11) Zhu, L.; Gamez, G.; Chen, H. W.; Huang, H. X.; Chingin, K.; Zenobi, R. Rapid Commun. Mass Spectrom. 2008, 22, 2993-2998.

(12) Gamez, G.; Zhu, L.; Disko, A.; Chen, H.; Azov, V.; Chingin, K.; Krämer, G.; Zenobi, R. Chem. Commun. 2011, 47, 4884-4886.

(13) Lento, C.; Wilson, D. J. Analyst 2017, 142, 1640-1653.

(14) Banerjee, S.; Zare, R. N. Angew. Chem. 2015, 127, 1500815012 .

(15) Girod, M.; Moyano, E.; Campbell, D. I.; Cooks, R. G. Chem. Sci. 2011, 2, 501-510.

(16) Perry, R. H.; Splendore, M.; Chien, A.; Davis, N. K.; Zare, R. N. Angew. Chem., Int. Ed. 2011, 50, 250-254.

(17) Bain, R. M.; Pulliam, C. J.; Thery, F.; Cooks, R. G. Angew. Chem. 2016, 128, 10634-10638.

(18) Loren, B. P.; Wleklinski, M.; Koswara, A.; Yammine, K.; Hu, Y.; Nagy, Z. K.; Thompson, D. H.; Cooks, R. G. Chem. Sci. 2017, 8, 4363-4370.

(19) Yan, X.; Cheng, H.; Zare, R. N. Angew. Chem., Int. Ed. 2017, 56, $3562-3565$.

(20) Badu-Tawiah, A. K.; Campbell, D. I.; Cooks, R. G. J. Am. Soc. Mass Spectrom. 2012, 23, 1461-1468.

(21) Wleklinski, M.; Falcone, C. E.; Loren, B. P.; Jaman, Z.; Iyer, K.; Ewan, H. S.; Hyun, S.-H.; Thompson, D. H.; Cooks, R. G. Eur. J. Org. Chem. 2016, 2016, 5480-5484.

(22) Müller, T.; Badu-Tawiah, A.; Cooks, R. G. Angew. Chem., Int. Ed. 2012, 51, 11832-11835.

(23) Cheng, S.; Wu, Q.; Xiao, H.; Chen, H. Anal. Chem. 2017, 89, 2338-2344.

(24) Waldner, M. J.; Neurath, M. F. Curr. Gene Ther. 2009, 9, 239247.

(25) Mann, S. Angew. Chem., Int. Ed. 2013, 52, 155-162.

(26) Michaud, V.; El Haddad, I.; Yao, L.; Sellegri, K.; Laj, P.; Villani, P.; Picard, D.; Marchand, N.; Monod, A. Atmos. Chem. Phys. 2009, 9, 5119-5130.

(27) Chen, H.; Cotte-Rodriguez, I.; Cooks, R. G. Chem. Commun. 2006, 597-599.

(28) Wu, C.; Qian, K.; Nefliu, M.; Cooks, R. G. J. Am. Soc. Mass Spectrom. 2010, 21, 261-267.

(29) Rao, W.; Mitchell, D.; Licence, P.; Barrett, D. A. Rapid Commun. Mass Spectrom. 2014, 28, 616-624.

(30) Nyadong, L.; Green, M. D.; De Jesus, V. R.; Newton, P. N.; Fernández, F. M. Anal. Chem. 2007, 79, 2150-2157.

(31) Huang, G.; Chen, H.; Zhang, X.; Cooks, R. G.; Ouyang, Z. Anal. Chem. 2007, 79, 8327-8332.

(32) Cotte-Rodríguez, I.; Takáts, Z.; Talaty, N.; Chen, H.; Cooks, R. G. Anal. Chem. 2005, 77, 6755-6764.

(33) Polenz, I.; Brosseau, Q.; Baret, J.-C. Soft Matter 2015, 11, 2916-2923.

(34) Gruner, P.; Riechers, B.; Semin, B.; Lim, J.; Johnston, A.; Short, K.; Baret, J.-C. Nat. Commun. 2016, 7, 10392.

(35) Rideout, D. C.; Breslow, R. J. Am. Chem. Soc. 1980, 102, 78167817.

(36) Narayan, S.; Muldoon, J.; Finn, M. G.; Fokin, V. V.; Kolb, H. C.; Sharpless, K. B. Angew. Chem., Int. Ed. 2005, 44, 3275-3279.

(37) Li, Y.; Yan, X.; Cooks, R. G. Angew. Chem. 2016, 128, 34943498.
(38) Lee, J. K.; Banerjee, S.; Nam, H. G.; Zare, R. N. Q. Rev. Biophys. 2015, 48, 437-444.

(39) Bain, R. M.; Pulliam, C. J.; Cooks, R. G. Chem. Sci. 2015, 6, 397-401.

(40) Takáts, Z.; Wiseman, J. M.; Gologan, B.; Cooks, R. G. Science 2004, 306, 471-473.

(41) Chen, H.; Venter, A.; Cooks, R. G. Chem. Commun. 2006, 2042-2044.

(42) Venter, A.; Sojka, P. E.; Cooks, R. G. Anal. Chem. 2006, 78, 8549.

(43) Lee, J. K.; Kim, S.; Nam, H. G.; Zare, R. N. Proc. Natl. Acad. Sci. U. S. A. 2015, 112, 3898-3903.

(44) Nemes, P.; Huang, H.; Vertes, A. Phys. Chem. Chem. Phys. 2012, 14, 2501-2507.

(45) Nazari, M.; Ekelöf, M.; Khodjaniyazova, S.; Elsen, N. L.; Williams, J. D.; Muddiman, D. C. Rapid Commun. Mass Spectrom. 2017, 31, 1868-1874.

(46) van Geenen, F. A. M. G.; Franssen, M. C. R.; Schotman, A. H. M.; Zuilhof, H.; Nielen, M. W. F. Anal. Chem. 2017, 89, 4031-4037.

(47) Nemes, P.; Barton, A. A.; Li, Y.; Vertes, A. Anal. Chem. 2008, $80,4575-4582$.

(48) Vertes, A.; Nemes, P.; Shrestha, B.; Barton, A. A.; Chen, Z.; Li, Y. Appl. Phys. A: Mater. Sci. Process. 2008, 93, 885-891.

(49) Sripadi, P.; Nazarian, J.; Hathout, Y.; Hoffman, E. P.; Vertes, A. Metabolomics 2009, 5, 263-276.

(50) Nemes, P.; Barton, A. A.; Vertes, A. Anal. Chem. 2009, 81, $6668-6675$

(51) Nemes, P.; Vertes, A. Methods Mol. Biol. 2010, 656, 159-171.

(52) Shrestha, B.; Vertes, A. Anal. Chem. 2009, 81, 8265-8271.

(53) Shrestha, B.; Vertes, A. J. Visualized Exp. 2010, No. 43, e2144.

(54) Shrestha, B.; Patt, J. M.; Vertes, A. Anal. Chem. 2011, 83, 2947-2955.

(55) Nemes, P.; Vertes, A. Anal. Chem. 2007, 79, 8098-8106.

(56) Liu, Y.; Miao, Z.; Lakshmanan, R.; Loo, R. R. O.; Loo, J. A.; Chen, H. Int. J. Mass Spectrom. 2012, 325-327, 161-166.

(57) Barbara, J. E.; Eyler, J. R.; Powell, D. H. Rapid Commun. Mass Spectrom. 2008, 22, 4121-4128.

(58) Lebeau, D.; Reiller, P. E.; Lamouroux, C. Talanta 2015, 132, $877-883$.

(59) Lostun, D.; Perez, C. J.; Licence, P.; Barrett, D. A.; Ifa, D. R. Anal. Chem. 2015, 87, 3286-3293.

(60) Shin, E.; Cha, S. Bull. Korean Chem. Soc. 2018, 39, 40-44.

(61) Nefliu, M.; Cooks, R. G.; Moore, C. J. Am. Soc. Mass Spectrom.

2006, 17, 1091-1095.

(62) Zhang, Y.; Chen, H. Int. J. Mass Spectrom. 2010, 289, 98-107.

(63) Wu, C.; Ifa, D. R.; Manicke, N. E.; Cooks, R. G. Anal. Chem. 2009, 81, 7618-7624.

(64) Song, Y.; Cooks, R. G. J. Mass Spectrom. 2007, 42, 1086-1092.

(65) Tian, Y.; Chen, J.; Ouyang, Y.; Qu, G.; Liu, A.; Wang, X.; Liu, C.; Shi, J.; Chen, H.; Jiang, G. Anal. Chim. Acta 2014, 814, 49-54.

(66) Ding, J.; Gu, H.; Yang, S.; Li, M.; Li, J.; Chen, H. Anal. Chem. 2009, 81, 8632-8638.

(67) Jiang, J.; Chen, S.; Li, M.; Li, H.; Chen, Y. Anal. Lett. 2017, 50, 797-805.

(68) Tang, F.; Guo, C.; Ma, X.; Zhang, J.; Su, Y.; Tian, R.; Shi, R.; Xia, Y.; Wang, X.; Ouyang, Z. Anal. Chem. 2018, 90, 5612-5619.

(69) Devenport, N. A.; Blenkhorn, D. J.; Weston, D. J.; Reynolds, J. C.; Creaser, C. S. Anal. Chem. 2014, 86, 357-361.

(70) Shrestha, B.; Nemes, P.; Nazarian, J.; Hathout, Y.; Hoffman, E. P.; Vertes, A. Analyst 2010, 135, 751-758.

(71) Nazari, M.; Malico, A. A.; Ekelöf, M.; Lund, S.; Williams, G. J.; Muddiman, D. C. Anal. Bioanal. Chem. 2018, 410, 953-962.

(72) Elliott, T. S.; Bianco, A.; Townsley, F. M.; Fried, S. D.; Chin, J. W. Cell Chem. Biol. 2016, 23, 805-815.

(73) Erdmann, R. S.; Takakura, H.; Thompson, A. D.; RiveraMolina, F.; Allgeyer, E. S.; Bewersdorf, J.; Toomre, D.; Schepartz, A. Angew. Chem., Int. Ed. 2014, 53, 10242-10246.

(74) Chen, W.; Wang, D.; Dai, C.; Hamelberg, D.; Wang, B. Chem. Commun. 2012, 48, 1736-1738. 
(75) Wang, D.; Chen, W.; Zheng, Y.; Dai, C.; Wang, K.; Ke, B.; Wang, B. Org. Biomol. Chem. 2014, 12, 3950-3955.

(76) Lang, K.; Davis, L.; Wallace, S.; Mahesh, M.; Cox, D. J.; Blackman, M. L.; Fox, J. M.; Chin, J. W. J. Am. Chem. Soc. 2012, 134, 10317-10320.

(77) Bruins, J. J.; Albada, B.; van Delft, F. Chem. - Eur. J. 2018, 24, $4749-4756$.

(78) Goor, O. J. G. M.; Brouns, J. E. P.; Dankers, P. Y. W. Polym. Chem. 2017, 8, 5228-5238.

(79) Sen, R.; Escorihuela, J.; van Delft, F.; Zuilhof, H. Angew. Chem., Int. Ed. 2017, 56, 3299-3303.

(80) Gahtory, D.; Sen, R.; Kuzmyn, A. R.; Escorihuela, J.; Zuilhof, H. Angew. Chem., Int. Ed. 2018, 57, 10118.

(81) Yan, X.; Bain, R. M.; Cooks, R. G. Angew. Chem., Int. Ed. 2016, $55,12960-12972$.

(82) Liu, P.; Zhang, J.; Ferguson, C. N.; Chen, H.; Loo, J. A. Anal. Chem. 2013, 85, 11966-11972. 